\title{
The Burden of Anemia Remains Significant over Time in Patients with Inflammatory Bowel Diseases at a Tertiary Referral Center
}

\author{
Alex Al Khoury ${ }^{1}$, Kelita Singh ${ }^{1}$, Zsuzsanna Kurti ${ }^{2}$, Lorant Gonczi ${ }^{2}$, Petra A. Golovics ${ }^{3}$, Rita Kohen ${ }^{1}$, Waqqas Afif ${ }^{1}$, Gary \\ Wild $^{1}$, Alain Bitton ${ }^{1}$, Talat Bessissow ${ }^{1}$, Peter Laszlo Lakatos ${ }^{1,2}$
}

1) McGill University,

Division of Gastroenterology

Department of Medicine,

Montreal, Canada;

2) Semmelweis University,

1st Department of Medicine,

Budapest, Hungary;

3) HDF Medical Centre,

Division of Gastroenterology, Budapest, Hungary.

\begin{abstract}
Background \& Aims: Anemia is a common complication of inflammatory bowel diseases (IBD), as well as a predictor of poor outcomes. The aim of this study was to determine the prevalence of anemia over time and the management of moderate to severe anemia at a tertiary referral IBD center.

Methods: We retrospectively reviewed the occurrence of anemia at the time of referral or diagnosis and during follow-up at the McGill University Health Centre IBD center. Consecutive patients presenting with an outpatient visit between July and December 2016 and between December 2018 and March 2019 were included. Disease characteristics, biochemistry and medical management, including the need for intravenous iron therapy were recorded.

Results: 1,356 Crohn's disease (CD) and 1,293 ulcerative colitis (UC) patients [disease duration: 12 (IQR: 6-22) and 10 (IQR: 5-19) years respectively] were included. The prevalence of moderate to severe anemia at referral/diagnosis (15.4\% and $8.5 \%)$ and during follow-up (11.1\% and $8.1 \%)$ were higher in CD than in UC patients. In CD, previous resective surgery, perianal disease and elevated C-reactive protein (CRP) at assessment, while in UC steroid therapy, an elevated CRP and fecal calprotectin at assessment were associated with anemia in a multivariate analysis. Anemia improved by $>2 \mathrm{~g} / \mathrm{dL}$ in $56.5 \%$ after $4-6$ weeks (intravenous iron dose $>1000 \mathrm{mg}$ in $87 \%$ of patients).

Conclusion: Anemia occurred frequently in this IBD cohort, at referral to the center and during follow-up, and contributes to the burden of IBD in referral populations. Most patients were assessed for anemia regularly and with accurate anemia workup; however, the targeted management of moderate to severe anemia was suboptimal.
\end{abstract}

Key words: anemia - ulcerative colitis - iron - Crohn's disease - inflammatory bowel diseases.

Abbreviations: CBC: complete blood count; CD: Crohn's disease; CRP: C-reactive protein; EIM: extraintestinal manifestation; FCAL: fecal calprotectin; GI: gastrointestinal; Hb: hemoglobin; IBD: inflammatory bowel diseases; IDA: iron deficiency anemia; IQR: inter quartile range; IV: intravenous; NAID: non-anemic iron deficiency; RUAM: RAND/UCLA Appropriateness Method; TNF: tumor necrosis factor; UC: ulcerative colitis; WHO: World Health Organization.

\section{INTRODUCTION}

Received: 18.06 .2020 Accepted: 19.10 .2020
Anemia is an important complication and/or extraintestinal manifestation (EIM) of inflammatory bowel diseases (IBD) as well as a predictor of poor patient outcomes. It is present in all IBD phenotypes and is an established marker of disease activity [1]. Importantly, anemia has been shown to affect patients' cognition, their ability to function at work and their overall quality of life [2]. It is also associated with a significant increase in health care expenditures $[3,4]$.

The two most common forms of anemia in IBD are iron deficiency anemia (IDA) and anemia of chronic disease [5]. In addition, an overlap of both forms of anemia has been reported [6]. A European meta-analysis by Filmann et al. [7], reported an overall prevalence of anemia of $27 \%$ for CD and $21 \%$ for UC. Furthermore, Vegh et al. [8], reported that anemia was a marker of disease progression in IBD and was associated with hospitalization and surgery in both UC and CD patients. This underscores the importance of screening for anemia in IBD patients [9]. Recommendations for screening for IDA in 
IBD are described by the 2015 European Crohn's and Colitis Organization (ECCO) guidelines [10] which include complete blood count $(\mathrm{CBC})$, serum ferritin, and C-reactive protein (CRP). For patients in remission or with mild disease, these should be performed every 6 to 12 months. In outpatients with active disease, these indices should be performed at least every 3 months [8]. The anemia workup includes red blood cell indices, mean corpuscular volume, differential blood cell count, serum ferritin, soluble transferrin receptor, and CRP concentration. Once corrected, monitoring for recurrent iron deficiency should be performed every 6-12 months [6].

The requirement for iron supplementation in IBD patients can be very high and intravenous (IV) iron therapy has been shown to be better tolerated and more effective than oral iron treatment in raising ferritin levels $[11,12]$. This has been demonstrated in multiple real-world studies using IV iron isomaltoside [13-15]. A recent systematic review and Bayesian network meta-analysis performed on the five eligible randomized, controlled trials with a total population of 1,143 patients has shown ferric carboxymaltose to be the most effective preparation for the treatment of IDA in IBD, followed by iron sucrose, iron isomaltoside and lastly oral iron [16]. Nevertheless, oral iron is effective in patients with IBD and may be used in those with mild anemia, a clinically inactive disease and in those not previously intolerant to oral iron [10]. Using the RAND/UCLA Appropriateness Method (RUAM), experts developed a treatment algorithm for anemia in IBD [17] which showed that adjustment of IBD medication without iron supplementation was appropriate in all patients with active disease and non-anemic iron deficiency (NAID). For instance, Lucendo et al. [18], demonstrated that controlling disease activity with anti-tumor necrosis factor (TNF)- $a$ therapy was significantly and independently associated with resolution of anemia in IBD, without the need for iron replacement therapy. Oral iron was mainly used in those with NAID and mildly anemic patients without disease activity. Intravenous iron was used in most other indications especially if the hemoglobin level was less than $10 \mathrm{~g} / \mathrm{dL}$ [19].

The aims of this study were to determine the prevalence of anemia over time, and to assess the management of moderate to severe anemia at a tertiary referral IBD center. The second objective was to assess the risk factors for anemia in IBD.

\section{METHODS}

We retrospectively reviewed the prevalence of anemia at the time of referral or diagnosis and during follow-up at the McGill University Health Centre (MUHC) IBD center. To this end, we evaluated consecutive patients presenting for an outpatient visit in two periods, between July and December 2016 and between December 2018 and March 2019. If a patient had multiple visits, we evaluated the first follow-up visit during the observation period. Management of moderate to severe anemia was monitored in the second cohort prospectively with regards to the need for IV iron therapy. The following data were captured: demographic variables, IBD-related surgery, medication history (5-aminosalicylates, systemic corticosteroids, immunomodulators, TNF- $\alpha$ inhibitors, ustekinumab, vedolizumab), anemia and biochemistry profile including the need for IV iron therapy in the second period. Disease activity was defined by assessment of the clinical note (Harvey-Bradshaw Index and Partial Mayo score). Disease extent for UC, as well as disease location and behavior for $\mathrm{CD}$, were defined according to the Montreal Classification [20]. Anemia was defined according to the World Health Organization (WHO) criteria as a hemoglobin $(\mathrm{Hb})$ level of less than $13 \mathrm{~g} / \mathrm{dL}$ in men and less than $12 \mathrm{~g} / \mathrm{dL}$ in non-pregnant females. Hemoglobin levels of 11-11.9 g/dL in non-pregnant females or 11-12.9 g/dL in males were defined as mild anemia and $8-10.9 \mathrm{~g} / \mathrm{dL}$ for both males and non-pregnant females as moderate according to the WHO [21]. The type of anemia was classified using the ferritin and CRP levels. The normal ranges for ferritin, CRP and fecal calprotectin (FCAL) were 23.9-366 $\mu \mathrm{g} / \mathrm{L}, 0-5.5 \mathrm{mg} / \mathrm{L}$ and $0-250 \mu \mathrm{g} / \mathrm{g}$ respectively. A CRP above 5.0 $\mathrm{mg} / \mathrm{L}$ and FCAL above $250 \mu \mathrm{g} / \mathrm{g}$ were defined as elevated. A ferritin level of less than $50 \mu \mathrm{g} / \mathrm{L}$ was used to define IDA [5] . We ruled out other gastrointestinal (GI) and non-GI causes of anaemia, and folate as well as vitamin B12 levels were measured in all patients. Intravenous iron sucrose (Venofer) at $300 \mathrm{mg}$ $(15 \mathrm{~mL})$ in $250 \mathrm{~mL}$ sodium chloride $0.9 \%$ infused over 2 hours was given to IBD patients with moderate to severe anemia. This was given weekly for 4 weeks. At our center, Venofer is the intravenous iron replacement therapy available on the pharmacy formulary; therefore, all of the patients who were prescribed IV iron replacement were prescribed Venofer.

The study was approved by the McGill University Health Centre Research Ethics Board (REB: REB\# 2017-3178 authorized for research 20 June 2017 and REB \# 2020-5579 authorized for research 24 April 2019). The research protocol conforms to ethical guidelines of the 1975 Declaration of Helsinki and local regulations.

Statistical analyses were performed using the Statistical Package for Social Sciences (SPSS 18.0; SPSS INC., Chicago, Illinois). All continuous data were expressed as mean \pm standard deviation or as a median with IQR. For univariate analysis, we used t-test and Wilcoxon rank-sum test for continuous variables and $\chi^{2}$ test for proportion of discrete variables. For multivariate analysis we used logistic regression analysis. Baseline parameters were examined using regression analysis. Prevalence rates were calculated for anemia at diagnosis and follow-up. A p value of $<0.05$ was regarded as statistically significant.

\section{RESULTS}

A total of 2,649 patients were included in the two study periods (CD, $\mathrm{n}=1,356$ and $\mathrm{UC}, \mathrm{n}=1,293)$ The median disease duration was 12 (IQR:6-22) and 10 (IQR: 5-19) years for CD and UC, respectively. The patient characteristics during follow-up are displayed in Table I. In the CD cohort, there were 267 patients (19.7\%) with L2 (colonic) disease and 678 patients (50.4\%) with L3 (ileocolonic) disease. The majority of patients in the CD group $(53.3 \%)$ had B1 (non-stricturing and non-penetrating) disease. In the UC group, the majority of patients (47.3\%) had extensive (E3) disease.

The prevalence of moderate to severe anemia at referral/ diagnosis was higher in CD patients with (15.4\%) compared with UC patients (8.5\%) in UC patients. During follow-up, this 
Table I. Patient characteristics

\begin{tabular}{lcc}
\hline & $\begin{array}{c}\text { Crohn's diseases } \\
\mathrm{n}=1,356\end{array}$ & $\begin{array}{c}\text { Ulcerative colitis } \\
\mathrm{n}=1,293\end{array}$ \\
\hline $\begin{array}{l}\text { Localization in CD (L1/2/3/4), \% } \\
\text { Extent in UC (E 1/2/3), \% }\end{array}$ & $29.5 / 19.7 / 50.4 / 6.5$ & $16.1 / 36.4 / 47.3$ \\
Behavior, (B 1/2/3), \% & $53.3 / 26.5 / 20.2$ & - \\
Perianal disease, \% & 25.3 & - \\
Severity (S 1/2/3), \% & - & $58.6 / 26.9 / 14.5$ \\
Resective surgery, \% & 26.8 & \\
Colectomy, \% & 4.1 & 6.6 , (pouch 4.1\%) \\
Ostomy, \% & 6.4 & 1.5 \\
Extraintestinal manifestations, \% & 38.1 & 27.2 \\
\hline
\end{tabular}

Montreal classification for Crohn's disease L1: ileon, L2: colon; L3: ileocolon; L4: upper gastrointestinal tract; $\mathrm{B} 1$ : nonstricturing, non penetrating; $\mathrm{B}$ : structuring; $\mathrm{B} 3$ : penetrating; Montreal classification for ulcerative colitis: S1: mild; S2: moderate; S3: severe; E1: ulcerative proctitis; E2:, left-sided colitis; E3: pancolitis

remained higher in CD patients with (11.1\%) compared to UC patients $(8.1 \%)$. The prevalence of anemia decreased between the two periods only in CD patients. The prevalence of any anemia at follow-up was $22.4 \%$ in CD and $18.7 \%$ in UC, with $82.7 \%$ of patients being tested at least once for anemia during a 6-month period.

The risk factors for anemia are presented in Table II (univariate analysis). We identified the following risk factors for anemia in UC patients: more extensive disease, treatment with steroids or biologics at the time of referral but not during follow-up, active disease, or an elevated FCAL at the time of assessment. In CD patients, the following parameters were associated with a greater risk of anemia: active disease, elevated CRP or FCAL at the time of assessment, with complicated disease, perianal involvement and previous resective surgery.

In a multivariate analysis, previous resective surgery ( $p=0.03$; OR: 1.70 ; 95\%CI: 1.06-2.74), perianal disease $(p=0.06$; OR: 2.12 ; 95\%CI: 0.98-4.67) and elevated CRP ( $\mathrm{p}=0.04$; OR: 2.23; 95\%CI: 1.03-4.83) at assessment were associated with the presence of anemia in CD patients. In a multivariate analysis, the need of steroids ( $\mathrm{p}=0.04$; OR: 4.55 ; 95\%CI: 1.08-19.2), an elevated CRP ( $p=0.02$; OR: 5.02; 95\%CI: 1.23-20.5) or FCAL $(\mathrm{p}=0.07$; OR: $2.50 ; 95 \% \mathrm{CI}: 0.93-6.76)$ at assessment were associated with anemia in UC patients.

Intravenous iron therapy was prescribed in 37 (46.8\%) from 79 patients with moderate or severe anemia; $72.3 \%$ were having active disease (CD: $65.2 \%$, steroid treatment $83.3 \%$, biological therapy: 78.6\%, elevated CRP: $97.8 \%$, elevated FCAL: $73.9 \%$ ) in the 2nd cohort.

A total percentage of $91.3 \%$ of patients receiving IV iron had an extensive evaluation of anemia pre- and post-therapy (CBC, ferritin, transferrin, transferrin saturation, vitamin B12, folate). Anemia improved by $>2 \mathrm{~g} / \mathrm{dL}$ in $56.5 \%$ after $4-6$ weeks (intravenous iron dose $>1000 \mathrm{mg}$ in $87 \%$ of patients) of treatment. Four patients required blood transfusion. There was a tendency of lower response to IV iron in CD ( $\mathrm{p}=0.08)$ and in patients with active disease $(\mathrm{p}=0.11)$, but improvement of anemia was $>2 \mathrm{~g} / \mathrm{dL}$ and was not associated with gender, disease behavior/extent, elevated CRP or FCAL.

\section{DISCUSSION}

In the present study, anemia was frequently observed in IBD patients at the time of referral as well as during the period of follow-up and underscores the burden of anemia

Table II. Factors associated with anemia in patients with inflammatory bowel diseases in univariate analysis

\begin{tabular}{|c|c|c|c|c|c|c|}
\hline & \multicolumn{3}{|c|}{ Crohn's Disease } & \multicolumn{3}{|c|}{ Ulcerative Colitis } \\
\hline & $\mathrm{p}$ & OR & $95 \% \mathrm{CI}$ & $\mathrm{p}$ & OR & $95 \% \mathrm{CI}$ \\
\hline Active disease & 0.01 & 1.59 & $1.12-2.25$ & 0.025 & 1.98 & $1.12-3.51$ \\
\hline Elevated CRP & 0.02 & 1.78 & $1.10-2.89$ & 0.002 & 2.96 & $1.52-5.76$ \\
\hline Elevated FCAL & 0.007 & 2.55 & $1.30-4.98$ & 0.052 & 2.25 & $0.99-5.16$ \\
\hline Disease extent & - & - & - & 0.04 & - & - \\
\hline Perianal disease & 0.02 & 1.78 & $1.13-2.79$ & - & - & - \\
\hline $\begin{array}{l}\text { Complicated disease: } \\
\text { strictures or fistulas }\end{array}$ & 0.06 & - & - & - & - & - \\
\hline $\begin{array}{l}\text { Previous resective } \\
\text { surgery }\end{array}$ & 0.001 & 2.03 & $1.36-3.03$ & - & - & - \\
\hline $\begin{array}{l}\text { Treatment with steroids } \\
\text { (during follow-up) }\end{array}$ & - & - & - & 0.001 & 5.16 & $2.33-11.40$ \\
\hline
\end{tabular}

CRP: C-reactive protein; FCAL: fecal calprotectin 
in IBD referral populations. The prevalence of moderate to severe anemia was higher in CD than UC at referral/diagnosis ( $15.4 \%$ vs. $8.5 \%)$ as well as during follow-up (11.1\% vs. $8.1 \%)$. Ulcerative colitis patients with more extensive disease, treated with steroids or biologics at the time of referral but not during follow-up, active disease, or an elevated FCAL at the time of assessment was associated with a higher risk of anemia. Crohn's disease patients with active disease, elevated CRP or FCAL at the time of assessment, with complicated disease, perianal involvement, previous resective surgery or colonic disease location, had a higher risk of anemia. Our findings are consistent with previous studies demonstrating that CD patients with stricturing disease behavior have an increased risk of anemia compared to CD patients with inflammatory phenotypes and that extensive UC was associated with an increased risk of anemia [1]. Lucendo et al. [22] showed that female gender was the strongest risk factor for anemia in both $\mathrm{CD}$ and UC followed by elevated CRP ( $\geq 2 \mathrm{mg} / \mathrm{dL})$.

The range of anemia prevalence in IBDs varies based on the type of study and subpopulation [23, 24]. Filmann et. al. [7], found that the overall prevalence of anemia was $27 \%$ for CD and 21\% for UC in European. Eriksson et al. [25] showed that their general population based IBD cohort had a prevalence of anemia of $22.6 \%$, with a higher incidence and prevalence of anemia in CD than in UC. Our results are similar to these findings, likely due to a high percentage of complex disease phenotype in CD. The IBSEN study [24] showed that the prevalence of anemia at 10 years from diagnosis was $13 \%$ in $\mathrm{CD}$ and $7.5 \%$ in UC, whereas in another population-based study from southern Sweden [26] the prevalence of anemia was 9\% in CD and 5\% in UC. However, the prevalence estimates in these two studies were based on hemoglobin levels that were calculated at the out-patient clinic at follow-up. The prevalence appeared to be higher in patients admitted to hospital (estimated 70\%) and in newly diagnosed patients (estimated 65\%), whereas the occurrence of anemia appeared to be lower in out-patients (around 20\%) and in patients with established IBD (around 35\%) [6, 23, 27, 28].

Anemia is a common extraintestinal manifestation of IBD and it is frequently overlooked as a complication of the disorder. It is important to recognize it as it has a negative outcome on patients' well-being and is a predictor of poor patient outcomes. Therefore, it is important to screen for it at diagnosis and to monitor for its presence at regular intervals. Most patients were assessed for anemia regularly and received accurate IV iron therapy. However, the targeted management of moderate to severe anemia was suboptimal. Targeted management should follow the treatment algorithm by the RUAM recommendations [17]. Patients with active disease should receive IV rather than oral iron, because dietary iron absorption is negatively affected by inflammation via the action of cytokines and the iron regulatory peptide, hepcidin $[29,30]$. One must keep in mind that IV iron therapy may increase the risk of infection compared to oral iron [31] although side effects (anaphylactic shock, bronchospasm, and hypotension) are rare [32]. In the present study, IV iron therapy was prescribed in less than half of patients with moderate or severe anemia, three quarters of whom had active disease. This highlights that the management of moderate to severe anemia was suboptimal even in this referral IBD cohort and more emphasis should be placed on anemia management as a part of quality of care indicators. In addition, $56.5 \%$ of the patients showed an improvement of anemia after 4-6 weeks of IV iron therapy, which suggests that patients with active disease may need higher doses or repeated IV iron administration.

The strengths of the present study include that data were obtained in a tertiary referral center with harmonized care protocols for the management of IBD and a strong emphasis on delivering quality care to these patients, yet it did not include a specific protocol of management of anemia in IBD patients. The consecutive inclusion of patients of all ages, comprising recently diagnosed IBD and long-standing IBD and living in a well-defined geographical area is an additional strength of this study. In addition, consecutive patients were included to avoid selection bias. The study has multiple limitations, including the retrospective nature of the study. The cohort represents realworld clinical practices and anemia screening was not regarded as a quality indicator in the center, thus adherence to harmonized guidelines were not followed during anemia work-up. Therefore, this study serves to identify an area in which our center may improve care by addressing iron deficiency anemia as a key quality indicator and defining a protocol for its management. Data on oral iron supplementation could not be tracked reliably. The data presented here are directly applicable to clinical practice, since they reinforce that harmonized screening and management of anemia in IBD centers during follow-up should form part of the mainstay of comprehensive management of these patients.

\section{CONCLUSIONS}

Anemia contributes to the burden of IBD in referral populations. The prevalence of anemia was high around the time of diagnosis and lower, but still significant during follow up in this referral IBD cohort, with a higher prevalence of anemia in CD compared to UC patients. Most patients were assessed for anemia regularly and with accurate anemia workup. However, IV iron therapy was underused, and only half of the patients reached target hemoglobin values. Thus, the management of moderate to severe anemia was suboptimal. The results from this study highlight the importance of anemia in IBD care and suggest the use of harmonized anemia assessment and management as a quality indicator in IBD centers to optimize the management of IBD patients.

Conflict of interest: None declared by all authors.

Authors' contributions: A.A. and P.L.L. conceived and designed the study. A.A. and K.S. collected the data. A.A., K.S. and P.L.L. analyzed the data. A.A. and K.S. drafted the manuscript. Z.K., L.G., P.G., R.K., W.A., G.W., A.B., and T.B. revised the article for intellectual content. All authors approved the final version of the submitted manuscript and agreed to be accountable for all aspects of the work.

\section{REFERENCES}

1. Burisch J, Vegh Z, Katsanos KH, et al. Occurrence of Anaemia in the First Year of Inflammatory Bowel Disease in a European Population- 
based Inception Cohort-An ECCO-EpiCom Study. J Crohns Colitis 2017;11:1213-1222. doi:10.1093/ecco-jcc/jjx077

2. Wells CW, Lewis S, Barton JR, Corbett S. Effects of changes in hemoglobin level on quality of life and cognitive function in inflammatory bowel disease patients. Inflamm Bowel Dis 2006;12:123130. doi:10.1097/01.MIB.0000196646.64615.db

3. Ershler WB, Chen K, Reyes EB, Dubois R. Economic burden of patients with anemia in selected diseases. Value Health 2005;8:629-638. doi:10.1111/j.1524-4733.2005.00058.x

4. Danese S, Hoffman C, Vel S, et al. Anaemia from a patient perspective in inflammatory bowel disease: results from the European Federation of Crohn's and Ulcerative Colitis Association's online survey Eur J Gastroenterol Hepatol 2014;26:1385-1391. doi:10.1097/ MEG.0000000000000200

5. Oustamanolakis P, Koutroubakis IE, Kouroumalis EA. Diagnosing anemia in inflammatory bowel disease: beyond the established markers. J Crohns Colitis 2011;5:381-391. doi:10.1016/j.crohns.2011.03.010

6. Gasche C, Lomer MC, Cavill I, Weiss G. Iron, anaemia, and inflammatory bowel diseases. Gut 2004;53:1190-1197. doi:10.1136/ gut.2003.035758

7. Filmann N, Rey J, Schneeweiss S, et al. Prevalence of anemia in inflammatory bowel diseases in european countries: a systematic review and individual patient data meta-analysis. Inflamm Bowel Dis 2014;20:936-945. doi:10.1097/01.MIB.0000442728.74340.fd

8. Vegh Z, Kurti Z, Gonczi L, et al. Association of extraintestinal manifestations and anaemia with disease outcomes in patients with inflammatory bowel disease. Scand J Gastroenterol 2016;51:848-854 doi: $10.3109 / 00365521.2016 .1140807$

9. Akhuemonkhan E, Parian A, Miller K, Hanauer S, Hutfless S. Prevalence and screening for anaemia in mild to moderate Crohn's disease and ulcerative colitis in the United States, 2010-2014. BMJ Open Gastroenterol 2017;4:e000155. doi:10.1136/bmjgast-2017-000155

10. Dignass AU, Gasche C, Bettenworth D, et al. European consensus on the diagnosis and management of iron deficiency and anaemia in inflammatory bowel diseases. J Crohns Colitis 2015;9:211-222. doi:10.1093/ecco-jcc/jju009

11. Lee TW, Kolber MR, Fedorak RN, van Zanten SV. Iron replacement therapy in inflammatory bowel disease patients with iron deficiency anemia: a systematic review and meta-analysis. J Crohns Colitis 2012;6:267-275. doi:10.1016/j.crohns.2011.09.010

12. Gasche C, Berstad A, Befrits R, et al. Guidelines on the diagnosis and management of iron deficiency and anemia in inflammatory bowel diseases. Inflamm Bowel Dis 2007;13:1545-1553. doi:10.1002/ibd.20285

13. Frigstad SO, Haaber A, Bajor A, et al. The NIMO Scandinavian Study: A Prospective Observational Study of Iron Isomaltoside Treatment in Patients with Iron Deficiency. Gastroenterol Res Pract 2017;2017:4585164. doi:10.1155/2017/4585164

14. Kearns J, Jacob S. N08 Real-world experience: Treatment of iron deficiency anaemia (IDA) with intravenous (IV) iron in inflammatory bowel disease. J Crohns Colitis 2019;13(Suppl 1):S560. doi:10.1093/ ecco-jcc/jjy222.996

15. Stein J, Walper A, Klemm W, Farrag K, Aksan A, Dignass A. Safety and efficacy of intravenous iron isomaltoside for correction of anaemia in patients with inflammatory bowel disease in everyday clinical practice. Scand J Gastroenterol 2018;53:1059-1065. doi:10.1080/00365521.2018.1498914

16. Aksan A, Ișık H, Radeke HH, Dignass A, Stein J. Systematic review with network meta-analysis: comparative efficacy and tolerability of different intravenous iron formulations for the treatment of iron deficiency anaemia in patients with inflammatory bowel disease Aliment Pharmacol Ther 2017;45:1303-1318. doi:10.1111/apt.14043

17. Reinisch W, Chowers Y, Danese S, et al. The management of iron deficiency in inflammatory bowel disease-an online tool developed by the RAND/UCLA appropriateness method. Aliment Pharmacol Ther 2013;38:1109-1118. doi:10.1111/apt.12493

18. Lucendo AJ, Roncero Ó, Serrano-Duenas MT, et al. Effects of antiTNF-alpha therapy on hemoglobin levels and anemia in patients with inflammatory bowel disease. Dig Liver Dis 2020;52:400-407. doi:10.1016/j.dld.2019.11.019

19. Muñoz M, Gómez-Ramírez S, García-Erce JA. Intravenous iron in inflammatory bowel disease. World J Gastroenterol 2009;15:4666-4674. doi:10.3748/wjg.15.4666

20. Silverberg MS, Satsangi J, Ahmad T, et al. Toward an integrated clinical, molecular and serological classification of inflammatory bowel disease: report of a Working Party of the 2005 Montreal World Congress of Gastroenterology. Can J Gastroenterol 2005;19 Suppl A:5A-36A. doi:10.1155/2005/269076

21. Cappellini MD, Motta I. Anemia in Clinical Practice-Definition and Classification: Does Hemoglobin Change With Aging? Semin Hematol 2015;52:261-269. doi:10.1053/j.seminhematol.2015.07.006

22. Lucendo AJ, Arias Á, Roncero Ó, et al. Anemia at the time of diagnosis of inflammatory bowel disease: Prevalence and associated factors in adolescent and adult patients. Dig Liver Dis 2017;49:405-411. doi:10.1016/j.dld.2016.12.005

23. Wilson A, Reyes E, Ofman J. Prevalence and outcomes of anemia in inflammatory bowel disease: a systematic review of the literature. Am J Med 2004;116 Suppl 7A:44S-49S. doi:10.1016/j.amjmed.2003.12.011

24. Høivik ML, Reinisch W, Cvancarova M, Moum B; IBSEN study group. Anaemia in inflammatory bowel disease: a population-based 10-year follow-up. Aliment Pharmacol Ther 2014;39:69-76. doi:10.1111/apt.12541

25. Eriksson C, Henriksson I, Brus O, et al. Incidence, prevalence and clinical outcome of anaemia in inflammatory bowel disease: a population-based cohort study. Aliment Pharmacol Ther 2018;48:638645. doi:10.1111/apt.14920

26. Rejler M, Tholstrup J, Andersson-Gäre B, Spångéus A. Low prevalence of anemia in inflammatory bowel disease: a population-based study in Sweden. Scand J Gastroenterol 2012;47:937-942. doi:10.3109/0036552 1.2012 .672590

27. Kulnigg S, Gasche C. Systematic review: managing anaemia in Crohn's disease. Aliment Pharmacol Ther 2006;24:1507-1523. doi:10.1111/ j.1365-2036.2006.03146.x

28. Gomollón F, Gisbert JP. Anemia and inflammatory bowel diseases. World J Gastroenterol 2009;15:4659-4665. doi:10.3748/wjg.15.4659

29. Theurl I, Aigner E, Theurl M, et al. Regulation of iron homeostasis in anemia of chronic disease and iron deficiency anemia: diagnostic and therapeutic implications. Blood 2009;113):5277-5286. doi:10.1182/ blood-2008-12-195651

30. Ganz T. Systemic iron homeostasis. Physiol Rev 2013;93:1721-1741. doi:10.1152/physrev.00008.2013

31. Litton E, Xiao J, Ho KM. Safety and efficacy of intravenous iron therapy in reducing requirement for allogeneic blood transfusion: systematic review and meta-analysis of randomised clinical trials. BMJ 2013;347:f4822. doi:10.1136/bmj.f4822

32. Akhuemonkhan E, Parian A, Carson KA, Hutfless S. Adverse Reactions After Intravenous Iron Infusion Among Inflammatory Bowel Disease Patients in the United States, 2010-2014. Inflamm Bowel Dis 2018;24:1801-1807. doi:10.1093/ibd/izy063 